Research in Astron. Astrophys. 2010 Vol. XX No. XX, 000-000

http://www.raa-journal.org http://www.iop.org/journals/raa

$\boldsymbol{R}$ esearch in

A stronomy and

A strophysics

Received [2010] [July] [7]; accepted [2010] [Sep] [22]

\title{
Contradiction between strong lensing statistics and a feedback solution to the cusp/core problem
}

\author{
Da-Ming Chen ${ }^{1}$ and Stacy McGaugh ${ }^{2}$ \\ 1 National Astronomical Observatories, Chinese Academy of Sciences, Beijing 100012, China; \\ cdm@nao.cas.cn \\ 2 Department of Astronomy, University of Maryland, College Park, MD 20742-2421, USA
}

\begin{abstract}
Standard cosmology has many successes on large scales, but faces some fundamental difficulties on small, galactic scales. One such difficulty is the cusp/core problem. High resolution observations of the rotation curves for dark matter dominated low surface brightness (LSB) galaxies imply that galactic dark matter halos have a density profile with a flat central core, whereas N-body structure formation simulations predict a divergent (cuspy) density profile at the center. It has been proposed that this problem can be resolved by stellar feedback driving turbulent gas motion that erases the initial cusp. However, strong gravitational lensing prefers a cuspy density profile for galactic halos. In this paper, we use the most recent high resolution observations of the rotation curves of LSB galaxies to fit the core size as a function of halo mass, and compare the resultant lensing probability to the observational results for the well defined combined sample of the Cosmic Lens All-Sky Survey (CLASS) and Jodrell Bank/Very Large Array Astrometric Survey (JVAS). The lensing probabilities based on such density profiles are too low to match the observed lensing in CLASS/JVAS. High baryon densities in the galaxies that dominate the lensing statistics can reconcile this discrepancy, but only if they steepen the mass profile rather than making it more shallow. This places contradictory demands upon the effects of baryons on the central mass profiles of galaxies.
\end{abstract}

Key words: cosmology: theory—dark matter—galaxies: halos—gravitational lensing: strong

In the standard cosmological model (known as $\Lambda \mathrm{CDM}$ ), the universe is dominated by invisible components called dark energy $(\Lambda)$ and cold dark matter $(\mathrm{CDM})$. The $\Lambda \mathrm{CDM}$ cosmology is very successful in explaining the cosmic microwave background and the formation of large scale structure. However, there are challenges to $\Lambda \mathrm{CDM}$ on smaller scales (Coles, 2005). Here we focus on the cusp/core problem (Navarro, Frenk \& White, 1997; Jing, 2000; Jing \& Suto, 2002; Navarro et al,, 2004; Li \& Chen, 2009) and whether proposed solutions to this problem can be consistent with the observed frequency of strong gravitational lensing.

One possible solution to the cusp/core problem is turbulence driven by stellar feedback during galaxy formation. If this process drives massive clumps of gas through the central regions of the first dark matter halos to form (Mashchenko et al., 2006, 2008), the central cusp may transform into a soft core. Once established, phase space arguments imply that the core should persist through subsequent mergers (Dehnen, 2005; Kazantzidis et al., 2006), leading to a final halo profile with a finite core radius for all galaxies, including giant ellipticals. Such a situation is consistent with essentially all kinematic 
Table 1 Halo Profiles

\begin{tabular}{lcc}
\hline Halo & $\gamma$ & $\rho(r)$ \\
\hline & & \\
SIS & 2 & $\rho_{0}\left(r / r_{0}\right)^{-2}$ \\
NFW & 1 & $\rho_{i}\left[\left(r / r_{s}\right)\left(1+r / r_{s}\right)^{2}\right]^{-1}$ \\
CIS & 0 & $\rho_{0}\left[1+\left(r / r_{c}\right)^{2}\right]^{-1}$ \\
\hline
\end{tabular}

observations (McGaugh, 2007; Romanowsky et al., 2003). The stellar feedback model is claimed to be 'universal' to all masses of galaxies, so it should be verified by observations of galaxies not only with low mass like dwarfs and LSBs, but of all masses, especially large mass galaxies like giant ellipticals. We show here that if stellar feedback solution to the cusp/core problem (arising from low mass LSB galaxies) is true, then it should also pass the tests of the observations of massive galaxies, in particular the observations of strong gravitational lensing. To do so, we extrapolate the core size-halo mass relation established from rotation curve data of low mass galaxies to massive ellipticals so that we can calculate the strong lensing probabilities.

Gravitational lensing provides a powerful tool to detect dark matter. The lensing efficiency is very sensitive to the slope $\gamma$ of the central mass density profile $\left(\rho \propto r^{-\gamma}\right)$. It is well established (Chae et al., 2002; Li \& Ostriker, 2002; Oguri et al., 2008) that when galaxies are modeled as a singular isothermal sphere (SIS: $\gamma=2$ ) and galaxy clusters are modeled as a Navarro-Frenk-White (NFW: $\gamma=1$ ) profile (see Table 1), the predicted strong lensing probabilities match the results from CLASS/JVAS. A steeper density slope near the center gives a more efficient lensing rate. For example, if we model galaxies with an NFW rather than SIS profile, the lensing probabilities are too low compared with observations at small image separations ( $\mathrm{Li} \&$ Ostriker, 2002). The presence of a central flat core $(\gamma \approx 0)$ in galaxies would further limit the lensing efficiency (Chen, 2005). For example, a nonsingular truncated isothermal sphere (NTIS), which is an analytical model (Shapiro et al., 1999) for the postcollapse equilibrium structure of virialized objects, has a soft core that matches quite well with the mass profiles of dark matter dominated LSB galaxies deduced from their observed rotation curves. The probabilities for lensing by NTIS halos are far too low compared to observations (Chen, 2005), however.

In order to investigate the effect of a central core on the strong lensing efficiency, we use the density profile of the halos directly constrained by observed rotation curves. These are well fit Begeman et al., 1991) by the cored isothermal sphere (CIS). The CIS halo has a finite core radius $r_{c}$ within which the density is constant $(\gamma=0)$. As well as providing a good description of the data, the CIS provides a reasonable proxy for the unwieldy NTIS profile. Initially, we consider lensing by the dark matter halo itself, and later consider the additional effects of the baryons.

The best objects for tracing the mass profile of the dominant dark matter component are LSB galaxies. In other galaxy types, the stellar mass can provide a non-negligible contribution to the rotation velocity at observed radii. This is not the case for LSB galaxies, whose diffuse disks remain dark matter dominated (de Blok \& McGaugh, 1997) down to small radii. These objects persistently suggest that dark matter halos possess approximately flat cores (de Blok et al., 2001) that are best fit with CIS halos (Figure11).

We use the most recent results (de Naray et al., 2008) from a sample of LSB galaxies for which rotation curves have been derived from high-resolution optical velocity fields. For each halo, we calculate the mass $M$ by integrating the CIS density profile to the radius $r_{200}$. This is the radius of a sphere within which the average mass density is 200 times the critical density of the universe, typically taken (Navarro, Frenk \& White, 1997) as the virial radius,

$$
\begin{aligned}
M & =M_{200}=\int_{0}^{r_{200}} 4 \pi \rho_{c i s}(r) r^{2} d r \\
& =4 \pi \rho_{0} r_{c}^{2}\left[r_{200}-r_{c} \arctan \left(r_{200} / r_{c}\right)\right]
\end{aligned}
$$




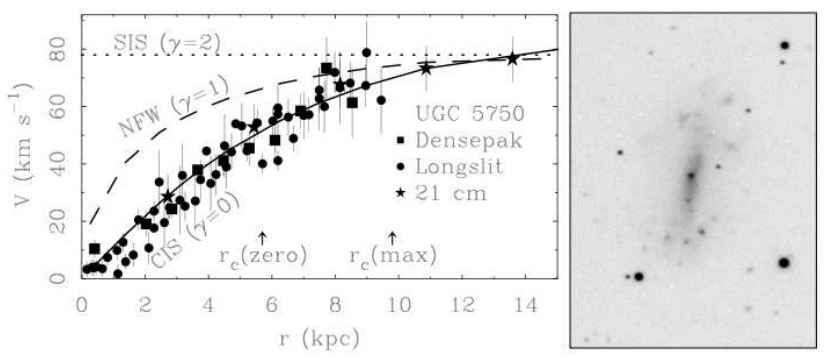

Fig. 1 The rotation curve (left) of the LSB galaxy UGC 5750 (right). Velocity data come from several independent sources and methods, including radio synthesis observations of the $21 \mathrm{~cm}$ spin flip transition of atomic hydrogen (van der Hulst et al., 1993), two independent (McGaugh et al., 2001; de Blok \& Bosma, 2002) optical long slit observations of the $n=3 \rightarrow 2$ Balmer transition $(\mathrm{H} \alpha)$, and Densepak integrated field $\mathrm{H} \alpha$ spectroscopy de Naray et al., 2006). The various halo types are shown as lines (as marked). The parameters of NFW halos are not free, following (Navarro, Frenk \& White, 1997; de Naray et al., 2006) from $\Lambda$ CDM cosmology. The difference between this and the data is the cusp/core problem. The core radius of the CIS fit is marked by arrows for the cases of zero and maximum disk. For clarity, the full CIS halo is only shown for the case of zero disk. Attributing mass to the stellar disk detracts from the velocity that can be attributed to dark matter (albeit not much in the case of LSB galaxies), increasing $r_{c}$ as shown and makes the discrepancy with the NFW prediction of $\Lambda$ CDM more serious. Under no circumstances can the halos of LSB galaxies be modeled by SIS.

. We compute the halo mass for two bracketing assumptions (de Naray et al., 2008) about the mass of the baryonic disk: zero disk, in which the mass of stars and gas is neglected, and maximum disk, which attributes the most mass possible to the stars without exceeding the observed rotation. The primary difference between these two cases is in the core radius inferred for the halo. As more mass is attributed to the stars, less dark matter is necessary at small radii. Consequently, $r_{c}$ grows with stellar mass.

There is an established correlation between $\rho_{0}$ and $r_{c}$ that can be fitted with a power-law formula Kormendy \& Freeman, 2004). Then together with $M_{200}=(4 \pi / 3) r_{200}^{3} \times 200 \times \rho_{\text {crit }}$, equation (1) can be numerically solved for any $M$ and the solution can be approximated by a power-law formula (Salucci et al., 2007). We do not fit $\rho_{0}$ and $r_{c}$, instead, for each halo of the sample, we substitute the corresponding $\rho_{0}$ and $r_{c}$ into equation (1) to numerically obtain $M$, then fit $r_{c}$ and $M$ with a powerlaw form. The results are similar for the two methods. Since our aim is to investigate the effect of the core radius on strong gravitational lensing efficiency, we fit the relation between $r_{c}$ and $M$ (Figure 2). As a check, we repeat the procedure with independent data (de Blok \& Bosma, 2002). The results are indistinguishable.

The gravitational lensing principle tells us that for any spherically symmetric density profile (here, a CIS halo), multiple images of a source can be produced only if the central convergence $\kappa_{c}$ is larger than unity (Schneider et al., 1992). The central convergence is a measure of the central surface mass density of the lensing halos. It is both mass and redshift dependent. For singular density profiles such as SIS and NFW, the central value is divergent, so $\kappa_{c}>1$ is always satisfied and multiple images can be produced by any mass. For density profiles with a finite soft core, however, the condition $\kappa_{c}>1$ imposes a minimum mass threshold to produce multiple images. For CIS halos (Chen, 2005), we have $\kappa_{c} \propto M^{2 / 3} / r_{c}$. The larger the core radius, the larger the mass needed to ensure $\kappa_{c}>1$. While both the zero and maximum disk cases give similar $r_{c}-M$ relations, the more conservative case is that with the smaller core radius for a given mass; other choices would produce less lensing. We thus use the formula 


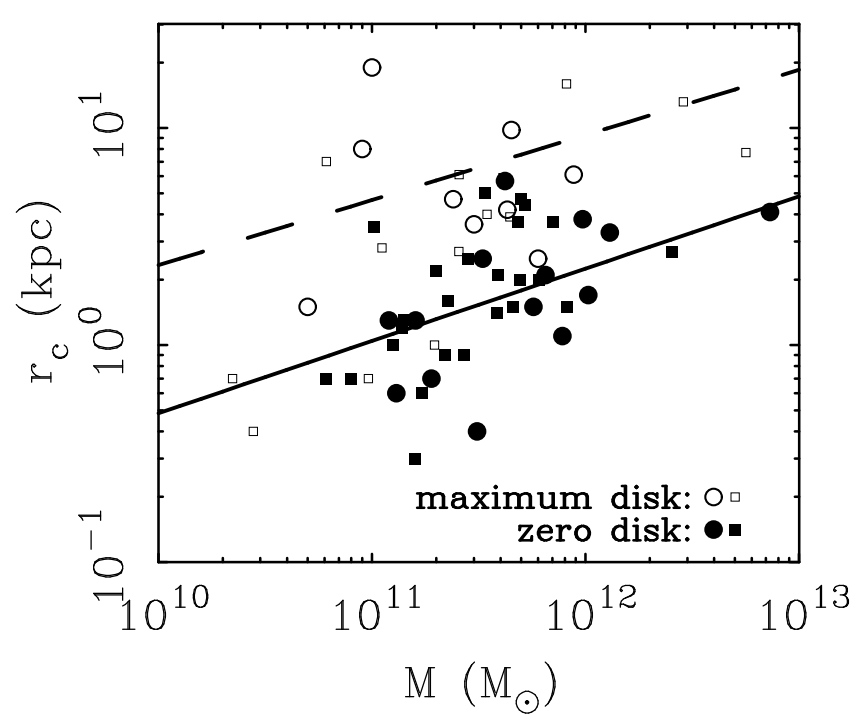

Fig. 2 The correlation between core radius $r_{c}$ and halo mass $M$. Filled points represent the case of zero disk mass and the open points represent maximum disk. The lines are the fit to the Densepak data (de Naray et al., 2008) only (circles); fits to long slit data (de Blok \& Bosma, 2002) (squares) give indistinguishable resuts.

fit to the zero disk case: $r_{c}=2.25\left(M / 10^{12} M_{\odot}\right)^{1 / 3} \mathrm{kpc}$. Interestingly, this formula is similar to the one derived analytically in the NTIS model (Shapiro et al., 1999; Chen, 2005).

The combined JVAS/CLASS survey forms a well-defined statistical sample containing 13 multiply imaged lens systems (Myers et al., 2003; Browne et al., 2003) among 8,958 sources. These data provide the image separation $\Delta \theta$ for each lens system. The observational probability $P_{\mathrm{obs}}(>\Delta \theta)$ for the CLASS/JVAS survey is shown in Figure 3 .

When a remote quasar is lensed by a CIS halo, three images are produced. The image nearest the lens is very weak. It disappears entirely when the source, lens, and observer are aligned, and the Einstein ring appears. The image separation $\Delta \theta$ is thus the separation between the outer two images. By adopting a model for the density profile of lensing halos, their comoving number density, and the geometry of the $\Lambda \mathrm{CDM}$ universe, we can predict the properties of the strong lens systems.

In order to compare with the observed lensing probabilities, we calculate $P_{\mathrm{CIS}}(>\Delta \theta)$, the lensing probability for quasars at redshift $z_{s}$ lensed by foreground CIS halos with image separation larger than $\Delta \theta$. The redshift $z_{s}$ of the sources (quasars) for the CLASS/JVAS sample has an approximately Gaussian distribution (Chae et al., 2002; Myers et al., 2003) with a mean of 1.27 and a dispersion of 0.95. The lensing rate is sensitive to $z_{s}$, but the effect of the redshift distribution is negligible compared to the choice of halo profile. We thus use the mean value $z_{s}=1.27$ in our calculations. For each lens system, the image separation depends on the source position. For the CIS model, however, the image separation is almost source position independent (Chen, 2005), so we use the diameter of the Einstein ring as the image separation for each lens system. Gravitational lensing magnifies the brightness of sources, so the number of lenses will be overrepresented (Turner et al., 1984) in any observed sample. The theoretically predicted lensing probability should therefore include a magnification bias (MB) correction to the observed probability. The MB is calculated on the basis of the total magnification of the outer two brighter images (Oguri et al., 2002). One of the most important elements in predicting lensing probability is the comoving number density of lensing galaxies. We adopt the results recently derived (Choi et al., 2007) from the Sloan Digital Sky Survey. The background cosmology is taken from the five-year Wilkinson Microwave Anisotropy Probe observations (Komatsu et al., 2009). The final predicted lensing probabil- 
ity for CIS is plotted in Figure 3 . For comparison, the lensing probability of the SIS model is shown with the same parameters and approximations as CIS. The NFW model (Chen, 2005) is also shown. This is important to modeling the lensing by clusters, but is not relevant on the scale of individual galaxies considered here.

The predicted lensing probability for the CIS modeled dark matter halos is about four orders of magnitude lower than the observations of CLASS/JVAS at all image separations, and two orders of magnitude lower than the NFW model at small image separations. Though successful in fitting rotation curves, the CIS model is obviously inadequate for explaining strong gravitational lensing. We have used a spherical model. As it is known that the ellipticity does not significantly affect the total lensing efficiency for SIS model (Huterer et al., 2005) when compared with the inner density slope on galaxy scales. This is in contrast to galaxy clusters, in which the main inner density slope (NFW like, $\gamma \sim 1)$ is shallower than SIS $(\gamma \sim 2)$ and thus ellipticity and substructures would significantly increase the lensing efficiency(Bartelmann et al., 1998; Meneghetti et al., 2001, 2003; Hennawi et al., 2007; Broadhurst \& Barkana, 2008). Similarly, for large core size CIS model $(\gamma \sim 0)$, lensing rate would become extremely more sensitive to the lens shape and to external perturbations. However, the combination of all our approximations together can shift the result by no more than one order of magnitude, as can be seen from the close match of our approximate SIS model to the data. So it is safe to conclude that dark halo models like CIS and NTIS with the soft central cores derived from kinematic observations can not account for the statistics of strong gravitational lensing by themselves; they need a more centrally concentrated component like the baryons.

It is not difficult to understand the low lensing probability of the CIS model. Recall that the central convergence depends on the mass $M$ and the redshift $z_{L}$ of the lensing halos. With the fitting formula $r_{c} \propto M^{1 / 3}$, we have $\kappa_{c}\left(M, z_{L}\right) \sim M^{1 / 3} F\left(z_{L}\right)$, where $F\left(z_{L}\right)=\Omega\left(z_{L}\right)^{1 / 6} D_{L} D_{L S} / D_{S}$, with $\Omega\left(z_{L}\right)=\Omega_{m}(1+$ $\left.z_{L}\right)^{3}+\Omega_{\Lambda}, D_{L}, D_{S}$ and $D_{L S}$ are the angular diameter distances from the observer to the lens, to the source and from the lens to the source, respectively. For quasars at $z_{S}=1.27, F\left(z_{L}\right)$ has a maximum value of 0.24 for $z_{L}$ in the interval $\left[0, z_{S}\right]$. The condition $\kappa_{c}>1$ for strong lensing implies $M>3 \times 10^{13} M_{\odot}$. Since $M \sim 10^{13} M_{\odot}$ corresponds to the most massive galaxies in the present universe, the galaxies with lower mass provide no contribution to the total lensing probability. Furthermore, the contributions of all galaxies to the total lensing probabilities are governed by the comoving number density $n(M)$, which has a high-mass exponential cutoff (Chen, 2008), $n(M) \sim \exp \left(-M^{\beta / 3}\right)$, with $\beta=2.67$ in our calculations. Consequently, galaxies with mass lower than $\sim 10^{13} M_{\odot}$ make no contribution, and highmass galaxies meet the exponential cutoff. Some previous work (Hinshaw \& Krauss, 1987; Kochanek, 1996; Chiba \& Yoshii, 1999) also used the CIS model for early-type galaxies to calculate the strong lensing probabilities, and obtained reasonable results. They adopted a typical value of the core radius of $r_{c} \sim 0.1 \mathrm{kpc}$, much smaller than ours $(\sim 2.25 \mathrm{kpc})$, so hardly different from SIS.

The only difference between CIS and SIS is that CIS has a finite core radius. While the SIS model matches the lensing observations quite well, the low lensing probabilities of the CIS model is in serious contradiction to observations of strong gravitational lensing. Similarly, the NFW/SIS model contradicts rotation curve data. The proposed remedy (Mashchenko et al., 2006, 2008) of the cusp/core problem via feedback driven turbulence fixes this problem at the expense of creating another.

Most lensing galaxies are giant elliptical galaxies with substantial stellar masses, while we base the CIS model on observations of dark matter dominated LSB galaxies. These are very different galaxy types. Lensing is not sensitive to whether the mass doing the lensing is baryonic or dark, so the contradiction might be avoided if the total mass distribution of ellipticals — stars plus dark matter — can be modeled as SIS spheres. The challenge then becomes a self-consistent understanding of the formation of all galaxy types.

In the context of the $\Lambda \mathrm{CDM}$ structure formation paradigm, the initial condition for galaxy formation is the NFW halo. Baryonic gas dissipates and settles to the center of the gravitational potential defined by the dark matter to form the visible galaxy. As the gas collapses, the potential must adjust to the rearrangement of mass. This process, commonly referred to as adiabatic contraction (Barnes \& White, 1984; Gnedin et al., 2004; Sellwood \& McGaugh, 2005), has the effect of steepening (Dubinski, 1994) the mass profile (increasing $\gamma$ ). Since the NFW halo is not adequate to explain lensing on its own (Chen, 


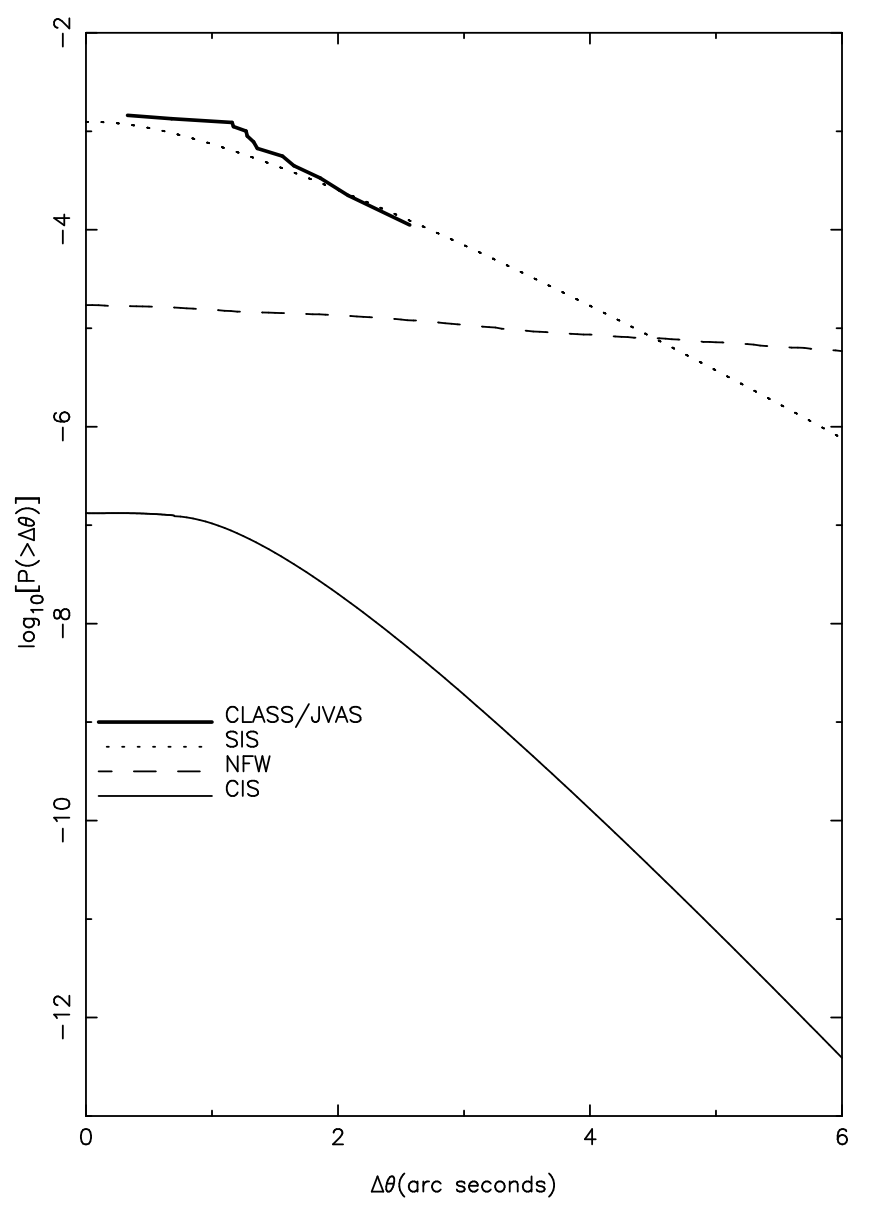

Fig. 3 The lensing probability with image separation larger than $\Delta \theta$. Our prediction for the CIS model based on the observed $r_{c}-M$ relation (Figure 2) is shown as the solid line. This fails to explain the observed lensing frequency (heavy line) by four orders of magnitude. In contrast, our approximate SIS model (dotted line) provides a reasonable match to the data. A pure NFW model (dashed line) gives intermediate results.

2005; Zhang, 2004) (Figure 3), this process seems necessary to produce elliptical galaxies that behave as SIS spheres. Indeed, any transformation other than $\gamma=1 \rightarrow 2$ would fail to reproduce the lensing statistics. However, this process cannot explain the observations of the rotation curves for LSB galaxies (Figure1).

In LSB galaxies, we need the opposite process: something that drives $\gamma$ from $1 \rightarrow 0$. This is what turbulence is proposed (Mashchenko et al., 2006, 2008) to do. The hypothesized turbulence is driven by feedback from early star formation in the first halos. If this process is universal and efficient, as proposed, then we may only solve the cusp/core problem at the expense of introducing a new problem with lensing. The baryons must first collapse to the center of the halo before they can drive feedback there. So only one process can dominate: either adiabatic contraction, which increases $\gamma$, or feedback, which might reduce $\gamma$. If feedback succeeds in establishing a soft core, it should persist through subsequent mergers (Dehnen, 2005; ; Kazantzidis et al,, 2006). It is difficult to see how an elliptical galaxy with an SIS mass profile could be constructed in this scenario. 
Nevertheless, this is what we need: dark halos with a soft core that persists in LSBs but elliptical galaxies that have a baryonic cusp. Observationally, there is no clear objection to having elliptical galaxies with a cuspy baryonic component embedded in a cored dark matter halo. The problem comes in self-consistenly building both kinds of galaxies.

Dark matter can only interact with baryons through gravity. The feedback of the baryons might re-shape the potential of the dark matter and then the total mass distribution. If strong outflows from stellar feedback carry dark matter particles out of the central region via gravity, when baryons cool and collapse to form the central baryonic cusp, they must necessarily bring back dark matter particles. The non-adiabatic action of sudden supernova driven outflows is only a minor perturbation on a zero sum game (Gnedin \& Zhao, 2002). In fact, recent simulations show that supernova-driven feedback inhibits the formation of baryonic bulges (cuspy baryons) and decrease the dark matter density (Governato et al, 2010), so that the total mass (baryons plus dark matter) density in the central regions of dwarf galaxies would be core-like rather than cusp-like. If this process is generically effective at producing cores in dark matter halos, then the early fragments that later build elliptical galaxies in $\Lambda \mathrm{CDM}$ should experience the same process. Indeed, there is considerably better evidence for strong star forming episodes elliptical galaxies than in LSBs. Once established, cores should persist through subsequent mergers in the entire mass distribution, both dark and baryonic.

We conclude that the apparent contradiction between rotation curves and strong lensing statistics pointed out here is genuine. It is difficult to simultaneously reconcile the soft cored halos favored by many kinematic observations with the singular mass profiles favored by strong lensing. In both cases, a fundamental tenet of the $\Lambda \mathrm{CDM}$ structure formation paradigm, the NFW halo, is inadequate to explain the observations. Substantial rearrangement of the initial NFW mass profile is required. Ideas hypothesized to solve one problem tend to make the other one worse.

Acknowledgements This work was supported by the National Natural Science Foundation of China under grant 11073023 and the National Basic Research Program of China (973 Program) under grant 2009CB24901, and by the National Science Foundation of the United States under grant AST0908370.

\section{References}

Barnes, J., \& White, S. D. M. 1984, MNRAS, 211, 753

Bartelmann, M., et al. 1998, A\&A, 330, 1

Begeman, K. G., Broeils, A. H., \& Sanders, R. H. 1991, MNRAS, 249, 523

Broadhurst, T. J., \& Barkana, R. 2008, MNRAS, 390, 1647

Browne, I. W. A., et al. 2003, MNRAS, 341, 13

Chae, K. -H., et al. 2002, Phys. Rev. Lett., 89, 151301

Chen, D. -M. 2005, ApJ, 629, 23

Chen, D.-M. 2008, J. Cosmol. Astropart. Phys., 01, 006

Chiba, M., \& Yoshii, Y. 1999, ApJ, 510, 42

Choi, Y. -Y., Park, C., \& Vogeley, M. S. 2007, ApJ, 658, 884

Coles, P. 2005, Nature, 433, 248

de Blok, W. J. G., \&Bosma, A. 2002, A\&A385, 816

de Blok, W. J. G., \& McGaugh, S. S. 1997, MNRAS, 290, 533

de Blok, W. J. G., McGaugh, S. S., Bosma, A., \&Rubin, V. C. 2001, ApJ, 552, L23

de Naray, R. Kuzio, McGaugh, S. S., \& de Blok, W. J. G. 2008, ApJ, 676, 920

de Naray, R. Kuzio, McGaugh, S. S., de Blok, W. J. G. \& Bosma, A. 2006, ApJS, 165, 461

Dehnen, W. 2005, MNRAS, 360, 892

Dubinski, J. 1994, ApJ, 431, 617

Gnedin, O. Y., Kravtsov, A. V., Klypin, A. A., \& Nagai, D. 2004, ApJ, 616, 16

Gnedin, O. Y., \& Zhao, H. 2002, MNRAS, 333, 299

Governato, F., et al. 2010, Nature, 463, 203

Hennawi, J. F., Dalal, N., Bode, P., \& Ostriker, J. P. 2007, ApJ, 654, 714 
Hinshaw, G., \& Krauss, L. M. 1987, ApJ, 320, 468

Huterer, D., Keeton, C. R., \& Ma, C.-P. 2005, ApJ, 624, 34

Jing, Y. 2000, ApJ, 535, 30

Jing, Y., \& Suto, Y. 2002, ApJ, 574, 538

Kazantzidis, S., Zentner, A. R., \& Kravtsov, A. V. 2006, ApJ, 641, 647

Kochanek, C. S. 1996, ApJ, 466, 638

E. Komatsu et al. 2009, ApJS, 180, 330

Kormendy, J., \& Freeman, K. C. 2004, Proceedings of IAU Symposium "Dark Matter in Galaxies”, Eds. S. Ryder et al., 220, 377

Li, N., \& Chen, D. -M. 2009, Research in Astron. Astrophys. (RAA), 9, 1173

Li, L. -X., \& Ostriker, J. P. 2002, ApJ, 566, 652

Mashchenko, S., Couchman, H. M. P., \&Wadsley, J. 2006, Nature, 442, 539

Mashchenko, S., Wadsley, J., \& Couchman, H. M. P. 2008 Science, 319, 174

McGaugh, S. S. et al. 2007, ApJ, 659, 149

McGaugh, S. S., Rubin, V.C., \& de Blok, W. J. G. 2001, AJ, 122, 2381

Meneghetti, M. et al. 2001, MNRAS, 325, 435

Meneghetti, M., Bartelmann, M, \& Moscardini, L. 2003, MNRAS, 340, 105

Myers, S. T., et al. 2003, MNRAS, 341, 1

Navarro, J. F., Frenk, C. S., \& White, S. D. M. 1997, ApJ, 490, 493

Navarro, J. F., et al. 2004, MNRAS, 349, 1039

Romanowsky, A. J., et al. 2003, Science, 301, 1696

Oguri, M., Taruya, A., Suto, Y., \& Turner, E. L. 2002, ApJ, 568, 488

Oguri, M., et al. 2008, AJ, 135, 512

Salucci, P., et al. 2007, MNRAS, 378, 41

Schneider, P., Ehlers, J., \& Falco, E. E. 1992, Gravitational lenses(Springer-Verlag, Berlin), p.230

Sellwood, J. A., \& McGaugh, S. S. 2005, ApJ, 634, 70

Shapiro, P. R., Iliew, I. T., \& Raga, A. C. 1999, MNRAS, 307, 203

Turner, E. L., Ostriker, J. P., \& Gott, J. R. 1984, ApJ, 284, 1

van der Hulst, J. M. et al. 1993, AJ, 106, 548

Zhang, T.-J. 2004, ApJ, 602, L5 